\title{
Pseudo-odontoma em um cão da pradaria de rabo preto (Cinomys ludovicianus)
}

\author{
Pseudo-odontoma in a black tail praire dog (Cinomys ludovicianus) \\ Gisele Guiomara Stein ${ }^{1} \&$ André da Silva Caríssimi²
}

\begin{abstract}
RESUMO
Pseudo-odontoma é uma doença displásica que afeta a origem dos dentes incisivos em cães da pradaria criados em cativeiro. Este relato descreveu um caso de pseudo-odontoma em um cão da pradaria de rabo preto, criado em cativeiro como animal de estimação no estado da Flórida, Estados Unidos. Uma fêmea de aproximadamente 4 anos de idade e 1,4 quilogramas, apresentava dificuldade respiratória e dispnéia. Não foram evidenciadas secreção nasal ou estertores durante a ausculta, como também não foram relatadas alterações comportamentais ou na ingestão de água e alimentos. Foi conduzido ao exame radiográfico, onde constatou-se o ápice dos dentes incisivos superiores irregular e hiperplásico, demonstrando obstrução de fluxo de ar nasal. O animal foi submetido à cirurgia para extração dos dentes incisivos, sendo realizada indução anestésica com propofol por via intravenosa e mantido sob plano anestésico com isofluorano. No período pós-operatório foram realizadas aplicações de antibióticos, antiinflamatórios e analgésicos, sendo necessária a alimentação forçada até que este retornasse a comer normalmente. Os dentes extraídos apresentavam-se com deformidade óssea em sua origem, caracterizando os pseudo-odontomas.
\end{abstract}

Descritores: Pseudo-Odontoma, Cães da Pradaria, Cinomys ludovicianus, Dentes.

\begin{abstract}
Pseudo-odontoma is displasic diseases that affect incisors teeth in captive prairie dogs. This case report described a case of pseudo-odontoma in a black tail prairie dog, kept in captivity in Florida State, USA. The around 4 years old weight 1,4 kilograms and presented difficulty to breath and dyspnea, didn't showing nasal secretion or stertor in auscult. There were no behavior, feeding or drink changes. The radiographic examinations were performed and could see irregularity and hyperplasic superior apical incisors teeth, suggesting upper airway obstruction. The animal went for surgery to extraction superior incisors teeth, by using intravenous anesthesic induction with propofol and manutention by isofluorane. For the postoperative time was administer antibiotics, anti-inflammatory, analgesic and was necessary assistance feeding until the animal started eats normally. The extracted teeth was affectec with apical deformities.
\end{abstract}

Keywords: Pseudo-Odontoma, Praire Dogs, Cinomys ludovicianus, Teeth.

${ }^{1}$ Mestranda, Programa de Pós-graduação em Ciências Veterinárias, Universidade Federal do Rio Grande do Sul (UFRGS). ${ }^{2}$ Setor de Animais de Biotério, Faculdade de Veterinária (UFRGS), Porto Alegre, RS/Brasil. CORRESPONDÊNCIA: G. G. Stein [gisele.stein@ufrgs.br]. 


\section{INTRODUÇÃO}

Cinomys ludovicianus são animais herbívoros monogástricos pertencentes à ordem Rodentia, a qual se caracteriza pelo crescimento contínuo dos dentes incisivos e presença de distema (espaço entre dentes incisivos e molares). Habitante natural do sul de Saskatchewan até norte do México, essa espécie é a mais tipicamente capturada para comércio e caça esportiva. São capturados ainda quando jovens, principalmente no Texas, para abastecerem o comércio de pets dos Estados Unidos e Japão [2].

Sua dentição é constituída de 22 dentes, sendo 4 incisivos, 2 superiores e 2 inferiores, com crescimento contínuo pela base e sem inervação, não possuindo dentes caninos. Devido ao crescimento contínuo dos dentes incisivos, necessitam, no cativeiro, de brinquedos e materiais que possam desgastá-los. Se esses não lhe são providenciados, os incisivos podem crescer muito e causar má-oclusão, podendo resultar em inabilidade de apreensão correta dos alimentos, dificuldade para comer, abscessos e até a morte. [3].

Pseudo-odontoma é uma doença displásica que afeta a origem dos dentes incisivos. Esta é uma doença comum encontrada em cães da pradaria criados em cativeiro [1]. A causa ainda não é bem conhecida, mas acredita-se que possa estar associada com a alimentação, problemas de má oclusão dos dentes incisivos e muitas vezes aparecendo em incisivos danificados por comportamentos esteriotipados em cativeiro, como é o caso de batidas sucessivas da arcada dentária superior nas gaiolas de ferro. Todos esses fatores podem contribuir para o surgimento dos pseudo-odontomas [2].

\section{RELATO DO CASO}

O objetivo desse relato foi descrever um caso de pseudo-odontoma em um cão da pradaria de rabo preto, mantido em cativeiro no estado da Flórida, Estados Unidos. O animal de 4 anos de idade e 1,4 quilogramas foi atendido no Broward Avian and Exotic Animal Hospital, Flórida, EUA . Constatou-se que o mesmo apresentava uma enorme dificuldade respiratória, dispnéia, porém, sem nenhum tipo de secreção nasal ou ocular. Outros parâmetros como ausculta cardíaca, palpação e visualização dentária com auxílio de otoscópio não demonstravam nenhuma anormalidade. Não foram relatadas alterações comportamentais ou na ingestão de água e alimentos. Radiografias do crânio foram realizadas e sugeridos exames laboratoriais (Tabela 1) para verificar a saúde geral do paciente. Os exames solicitados se encontravam dentro dos parâmetros normais para a espécie.

Constatou-se, ao exame radiográfico, que o ápice dos dentes incisivos superiores estava irregular, hiperplásico e também que o tamanho da cavidade nasal estava reduzido (Figura 1), resultando em uma dificuldade na passagem de ar, fazendo com que o animal respondesse com severa dificuldade respiratória.

O paciente do caso aqui relatado foi submetido à cirurgia no dia seguinte. No pré-operatório, o animal recebeu propofol ${ }^{1}$ por via intravenosa como fármaco indutor e logo após procedeu-se a intubação do animal com auxílio de endoscópio devido à dificuldade de visualização da glote nesses animais, e foi mantido sob plano anestésico com isofluorano. ${ }^{2}$

Tabela 1. Resultados dos exames laboratoriais com valores de referências para Cinomys ludovicianus. Exames realizados no Broward Avian and Exotic Animal Hospital, Florida (USA), no mês de setembro do ano de 2006.

\begin{tabular}{|l|c|c|}
\hline & Caso Clínico & $\begin{array}{c}\text { Valores de } \\
\text { referência* }\end{array}$ \\
\hline Hematócrito (\%) & 48 & $36-54$ \\
\hline Proteína Plasmática Total (g/dL) & 8,2 & $5,8-8,1$ \\
\hline Fosfatase Alcalina (UI/L) & 52 & $25-64$ \\
\hline Alanina Aminotransferase (UI/L) & 10 & $26-91$ \\
\hline Nitrogênio uréico sanguíneo (mg/dL) & 12 & $21-44$ \\
\hline Creatinina (mg/dL) & 1 & $0,8-2,3$ \\
\hline
\end{tabular}

*Valores de referência [2]. 


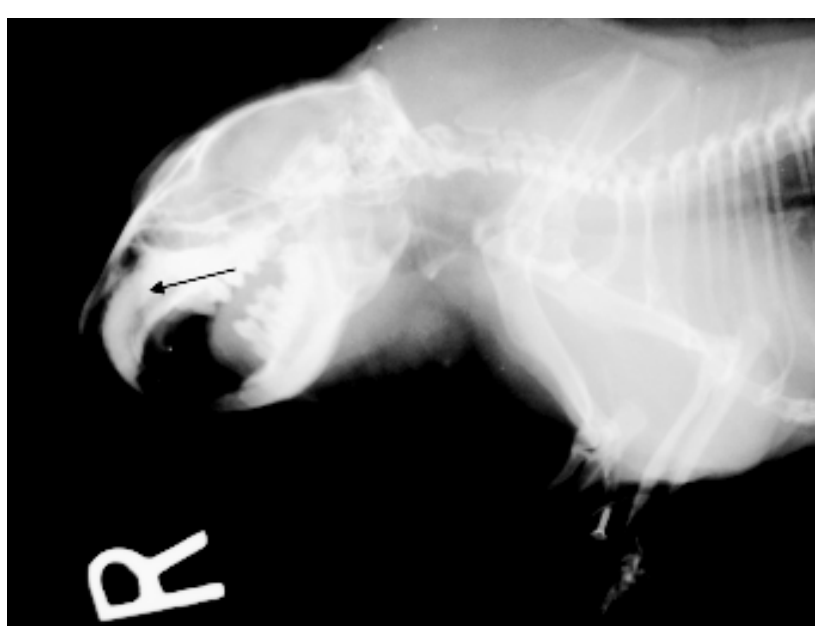

Figura 1. Ápice dos dentes incisivos superiores de Cinomys ludovicianus irregulares e hiperplásicos (seta) causando uma obstrução do fluxo de ar na cavidade nasal.

A extração dos incisivos superiores foi realizada observando-se uma grande deformação na origem destes dentes (Figura 2). No pós-operatório, o animal recebeu butorfanol ${ }^{3}$ subcutâneo $(0,1 \mathrm{mg} / \mathrm{Kg})$ TID, durante as primeiras 24 horas, enrofloxacina ${ }^{4}$ via oral $(5 \mathrm{mg} / \mathrm{Kg}) \mathrm{BID}$, durante 7 dias como tratamento profilático a possíveis infecções secundárias e meloxicam ${ }^{5}$ via oral $(0,2 \mathrm{mg} / \mathrm{Kg}) \mathrm{BID}$, durante duas semanas.

Foi prescrita alimentação forçada com Critical Care', TID. No terceiro dia após a cirurgia, o animal já apresentava uma melhora no desconforto respiratório e iniciou a comer sozinho.

\section{DISCUSSÃO}

Os primeiros sinais clínicos da doença são dispnéia, ruídos nasais, parcial ou completa obstrução do fluxo de ar, respiração pela boca (o animal aparece com a boca aberta) e ocasionalmente descarga nasal. O exame também mostra que os incisivos superiores são normalmente curtos, indicando que os animais possuem a tendência de morderem materiais duros ou algum outro trauma [5]. No caso desta paciente, os sinais clínicos levaram à suspeita da doença.

Mudanças anatômicas precoces incluem anormalidades e irregularidades da nova dentina. Se a erupção do dente tiver cessado, de qualquer modo, o resultado é uma severa deformação na origem, na qual a ação é uma massa ocupando um espaço e liderando uma progressiva obstrução nasal. A massa formada não é uma neoplasia verdadeira e, portanto,

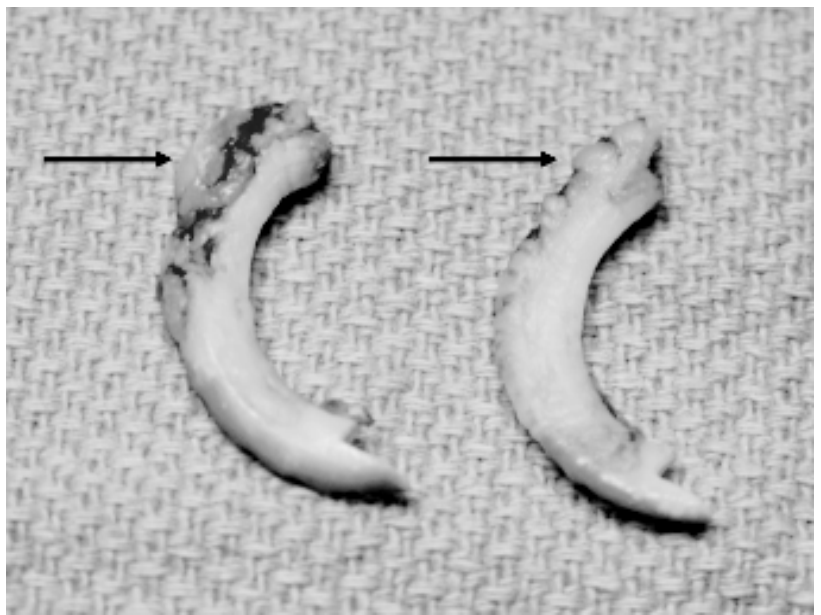

Figura 2. Dentes incisivos superiores de C. ludovicianus após extração cirúrgica, mostrando severa deformação (setas) na origem dos mesmos.

o termo "odontoma" é incorreto. Nas radiografias observa-se o ápice dos incisivos superiores irregulares e hiperplásicos. O tamanho da cavidade nasal é reduzido e a passagem de ar é limitada, resultando em dispnéia e espirro [2]. Como visto na radiografia relatada, a paciente apresentava uma diminuição significativa na passagem de ar da cavidade nasal, fazendo com que essa se tornasse dispnéica.

Tratamentos com antibióticos, descongestionantes e esteróides são paliativos [4]. Devido ao fato de tratamento medicamentoso não ter um efeito curativo, foi sugerida a extração dos dentes incisivos superiores para um melhor alívio dos sinais clínicos.

Após a cirurgia, os cães da pradaria convivem com boa saúde e os sinais clínicos não são recorrentes [1]. Um mês após a cirurgia, o animal apresentava uma melhora significativa, não apresentando mais nenhum sinal de dificuldade respiratória. Como profilaxia, foi sugerida a introdução de feno como alimentação para manutenção do desgastes dos incisivos inferiores.

\section{NOTAS INFORMATIVAS}

${ }^{1}$ Diprivan ${ }^{\circledR}$ - AstraZeneca - Wilmington, DE, Estados Unidos da América.

${ }^{2}$ Isosol@ - Vedco - St. Joseph, MO, Estados Unidos da América.

${ }^{3}$ Torbujesic ${ }^{\circledR}$ - Fort Dodge - Fort Dodge, IA, Estados Unidos da América.

${ }^{4}$ Baytril ${ }^{\circledR}$ - Bayer - Newbury, Reino Unido.

${ }^{5}$ Meloxicam ${ }^{\circledR}$ - Boehringer Ingelheim - Ridgefield, CT, Estados Unidos da América.

${ }^{6}$ Critical Care for Herbivorous - Oxbow - Murdock, NE, Estados Unidos da América. 


\section{REFERÊNCIAS}

1 Capello V. \& Gracis M. 2005. Rabbit and rodent dentistry handbook. Lake Worth: Zoological Eduaction Network, 276p.

2 Quesemberry K.E. \& Carpenter J.W. 2003. Ferrets, rabits, and rodents: clinical medicine and surgery. 2nd edn. St. Louis: Saunders, 461p.

3 Store P. 1995. Praire Dog pets. 2nd edn. Columbus: Country Storer Enterprises, 191p.

4 Wagner R.A., Garman R.H. \& Collins B.M. 1999. Diagnosing odontomas in praire dog. Exotic DVM Veterinary Magazine. 1: 7-10.

5 Wagner R.A. \& Jahnson D. 2001. Rhinotomy for treatment of odontoma in praire dog. Exotic DVM Veterinary Magazine. 3: 29.

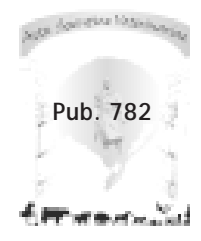

